\title{
ÜBER MEROMORPHE FUNKTIONEN MIT EINEM EXISTENZGEBIETE, DESSEN RAND EINE CANTOR'SCHE PUNKTMENGE VON DER KAPAZITÄT NULL IST
}

\author{
ÅGOT E. RIIBER
}

\section{Einleitung}

1. G. af Hällström [2] hat gezeigt, dass die in der Theorie der meromorphen Funktionen wohlbekannten Hauptsätze von Nevanlinna in modifizierter Form auch für solche eindeutige analytische Funktionen gelten, die in gewissen mehrfach zusammenhängenden Gebieten von meromorpher Natur sind (vgl. auch M. Tsuji [9][10] und K. Noshiro [6]).

Die Nevanlinna'schen Hauptsätze enthalten bekanntlich eine Aussage über die Beziehungen zwischen dem Verhalten der gegebenen Funktion $f(z)$ auf dem Kreise $|z|=r$ und deren Wertverteilung innerhalb desselben Kreises. Dieser Kreis kann als Niveaukurve der harmonischen Funktion $\ln |z|$ angesehen werden. Bei der Hällström'schen Erweiterung der Sätze übernehmen die Niveaukurven anderer harmonischer Funktionen die Rolle dieser Kreise. Wenn der Rand des Existenzgebietes eine Punktmenge von der Kapazität Null ist, wird von einer Potentialfunktion, die G. Evans [1] und H. Selberg [8] eingeführt haben, Gebrauch gemacht.

af Hällström beweist in seiner Abhandlung erst die Hauptsätze und untersucht dann eingehend zwei Spezialfälle: 1) Das Existenzgebiet der betrachteten analytischen Funktion ist die in endlich vielen Punkten punktierte Vollebene. 2) Es hat eine abzählbar unendliche Menge von Randpunkten mit einem einzigen Häufungspunkt.

Vorliegende Arbeit handelt von solchen analytischen Funktionen, deren Randpunkte eine verallgemeinerte Cantor'sche Menge von der Kapazität Null bilden. Zuerst wird eine Abschätzung des in dem zweiten Hauptsatze auftretenden Restgliedes gegeben, und danach werden einige hinreichende Bedingungen für die Gültigkeit der Defektrelation aufgestellt.

Ich bin Herrn Professor Ernst Jacobsthal für wertvolle Hilfe bei der Durchsicht des Manuskriptes grossen Dank schuldig.

Eingegangen am 30. Juni 1955. 


\section{Die Hauptsätze}

2. Dieses Kapitel enthält diejenigen Resultate von Hällström [2, Kap. I und II], die im folgenden gebraucht werden.

In der Einleitung wurde erwähnt, dass für analytische Funktionen mit mehrfach zusammenhängenden Existenzgebieten die Niveaukurven gewisser harmonischer Funktionen die Rolle der Kreise in der klassischen Theorie übernehmen. Wenn der Rand eine Punktmenge von der Kapazität Null ist, wird die Evans-Selberg'sche harmonische Funktion von Bedeutung. Es gilt:

Zu jeder abgeschlossenen beschränkten Punktmenge (a) der Kapazität Null gibt es eine Funktion $g(z)$ mit folgenden Eigenschaften:

$1^{\circ}$ In der Umgebung von $z=\infty$ unterscheidet sie sich von $-\ln |z|$ um eine harmonische Funktion.

$2^{\circ}$ Im Komplementärbereich $B$ von (a) ist sie sonst überall harmonisch.

$3^{\circ}$ Bei Annäherung an die a-Menge wächst $g(z)$ gleichmässig über alle Grenzen.

Sei nun $w=f(z)$ eine eindeutige analytische Funktion in einem Gebiete $B$, dessen Rand (a) von der eben genannten Art ist. Die Randpunkte werden als wesentliche Singularitäten von $f(z)$ vorausgesetzt. Sei $g(z)$ eine zu diesem Gebiete gehörige harmonische Funktion mit den Eigenschaften $1^{\circ}, 2^{\circ}$ und $3^{\circ}(g(z)$ ist im allgemeinen nicht eindeutig bestimmt). Wir denken uns in den Bereich $B$ die Niveaukurve

$$
G_{\lambda}: \quad g(z)=\lambda
$$

eingezeichnet. Eine solche Niveaukurve kann aus mehreren separaten geschlossenen Kurven bestehen. Wenn $\lambda$ von $-\infty$ bis $+\infty$ wächst, überstreicht $G_{\lambda}$ den ganzen Bereich $B$.

Im Inneren eines geschlossenen Teiles einer Niveaukurve $G_{\lambda}$ liegt notwendig ein Randpunkt von $B$. Denn $g(z)$ kann in einem solchen Gebiete wegen des Prinzips vom Maximum und Minimum nicht überall regulär sein.

3. Die nichtnegative Schmiegungsfunktion wird durch

$$
m(\lambda, w)=\frac{1}{2 \pi} \int_{G_{\lambda}} \ln (1 /[f(z), w]) d h
$$

definiert, worin

$$
\begin{gathered}
{\left[w_{1}, w\right]=\left|w_{1}-w\right|\left\{\left(1+\left|w_{1}\right|^{2}\right)\left(1+|w|^{2}\right)\right\}^{-\frac{1}{2}} \leqq 1,} \\
{\left[w_{1}, \infty\right]=\left(1+\left|w_{1}\right|^{2}\right)^{-\frac{1}{2}},}
\end{gathered}
$$

und $h(z)$ eine zu $g(z)$ konjugierte harmonische Funktion ist. 
Die Anzahlfunktion ist

$$
N(\lambda, w)=\int_{-\infty}^{\lambda} n(g, w) d g+\ln [f(\infty), w] .
$$

In diesem Ausdrucke bezeichnet $n(\lambda, w)$ die Anzahl der $w$-Stellen (mit richtiger Vielfachheit) im Gebiete $-\infty<g(z) \leqq \lambda$. Ist $z=\infty$ eine $n_{0}$-fache $w$-Stelle, so muss (2) durch

$$
N(\lambda, w)=\int_{-\infty}^{\lambda} n(g, w) d g+n_{0} \lambda+k
$$

ersetzt werden. Hier ist $k$ eine passend gewählte Konstante.

Über diese Grössen besagt der

Erste Hauptsatz. Die Summe der Schmiegungsfunktion und der Anzahlfunktion ist unabhängig von w. Die charakteristische Funktion

$$
T(\lambda)=m(\lambda, w)+N(\lambda, w)
$$

ist eine positive, wachsende, konvexe Funktion von $\lambda$.

4. Ferner gilt der

Zweite Hauptsatz. Sind $w_{1}, w_{2}, \ldots, w_{q}$ beliebige Zahlen, so ist

$$
\sum_{i=1}^{q} m\left(\lambda, w_{i}\right)+N_{1}(\lambda)<2 T(\lambda)+F(\lambda)+O(\ln T(\lambda))
$$

ausser vielleicht auf Intervallen, wo $\int T(\lambda) d \lambda$ endlich ist. Die Grösse $N_{1}(\lambda)$ ist die Anzahlfunktion der Verzweigungszahlen, die zu der dem Gebiete $g(z) \leqq \lambda$ entsprechenden $w$-Fläche gehören ${ }^{1}$, und $F(\lambda)$ ist ein Restglied.

Für $F(\lambda)$ gilt die Darstellung

$$
F(\lambda)=\int_{-\infty}^{\lambda} n_{G}(g) d g-\lambda+K,
$$

worin $n_{G}(\lambda)$ um eine Einheit kleiner ist als die Anzahl der Kurven, aus denen $G_{\lambda}$ besteht, und $K$ eine Konstante bedeutet. Die Funktion $n_{G}(\lambda)$ wächst monoton mit $\lambda$.

5. Der Defekt $\delta(w)$ wird für jedes $w$ durch

$$
\delta(w)=\liminf _{\lambda \rightarrow \infty}(m(\lambda, w) / T(\lambda))
$$

1 Siehe [2, S. 26]. 
und der Verzweigungsindex $\mu$ durch

$$
\mu=\liminf _{\lambda \rightarrow \infty}\left(N_{1}(\lambda) / T(\lambda)\right)
$$

eingeführt. Wenn $\lim _{\lambda \rightarrow \infty}(F(\lambda) / T(\lambda))=0$ ist, ergibt sich aus (4) die $D e$ fektrelation

$$
\sum_{i=1}^{q} \delta\left(w_{i}\right)+\mu \leqq 2
$$

\section{Der Fall, wo der Rand des Gebietes $B$ eine verallgemeinerte Cantor'sche Punktmenge von der Kapazität Null ist}

\section{§ 1. Die Potentialfunktion}

6. In einer nicht im Druck veröffentlichten Examensarbeit von O. Midtbö (Oslo, 1939) ist eine Punktmenge beschrieben, welche durch das folgende Konstruktionsprinzip erklärt wird:

Wir gehen von einem abgeschlossenen regulären $N$-eck $P_{0}(N \geqq 2)$ aus, dessen Seite $\Delta_{0}=1$ ist. Die Menge der Punkte von $P_{0}$ nennen wir

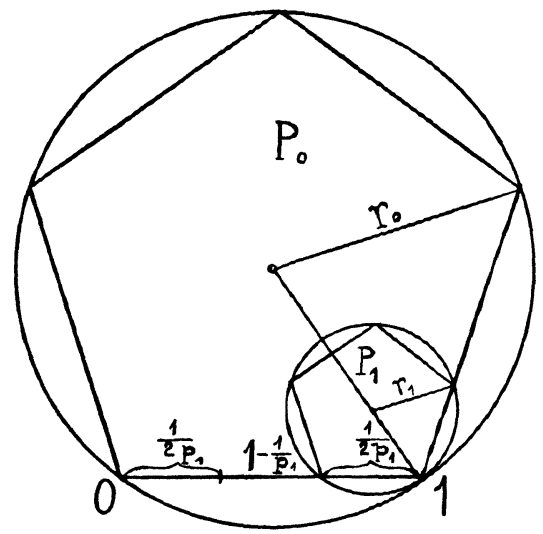

Fig. 1 .

$M\left(p_{0}\right)$. Aus jeder Seite entfernen wir eine offene Strecke von der Länge $1-1 / p_{1}\left(p_{1}>1\right)$, so dass beiderseits eine abgeschlossene Teilstrecke $\Delta_{1}$ von der Länge $1 /\left(2 p_{1}\right)$ übrig bleibt (siehe Fig. 1). In jeder Ecke von $P_{0}$ konstruieren wir nach innen ein abgeschlossenes regelmässiges $N$-eck von der Seitenlänge $1 /\left(2 p_{1}\right)$. Jedes der neuen $N$-ecke heisse $P_{1}$. Wir sagen, sie seien durch die Operation $\left(p_{1}\right)$ aus $P_{0}$ hervorgegangen. Die 
Vereinigungsmenge ihrer Punkte bezeichnen wir mit $M\left(p_{0} p_{1}\right)$. Auf jedes $N$-eck $P_{1}$ wird nun die Operation $\left(p_{2}\right)$ angewendet u. s. w. Nach den $n$ Operationen $\left(p_{1}\right),\left(p_{2}\right), \ldots,\left(p_{n}\right)$ haben wir im ganzen $N^{n}$ regelmässige $N$-ecke $P_{n}$ bekommen, deren Punkte die Menge $M\left(p_{0} p_{1} \ldots p_{n}\right)$ ausmachen. Dieser Prozess wird nun unbeschränkt fortgesetzt. Die Seite eines $\operatorname{der} P_{n}$ nennen wir $\Delta_{n}$, und der Umkreis heisse $C_{n}$. Er hat den Radius $r_{n}$ und den Durchmesser $d_{n}$. Wir sagen, zwei $C_{n}$ seien zu einander assoziiert, wenn sie in demselben $C_{n-1}$ liegen. Es sei

$$
p=\inf p_{n}>\frac{1}{2}+\frac{1}{2 \sin (\pi / N)} \quad(n=1,2, \ldots),
$$

was eine hinreichende Bedingung dafür ist, dass zwei assoziierte $C_{n}$ punktfremd sind. Den abgeschlossenen Durchschnitt $M\left(p_{0} p_{1} \ldots\right)$ von $M\left(p_{0}\right), M\left(p_{0} p_{1}\right), M\left(p_{0} p_{1} p_{2}\right), \ldots$ hat Midtbö eine verallgemeinerte Cantor'sche Punktmenge $N$-ter Ordnung genannt. Für $N=2$ erhalten wir die lineare Cantor'sche Menge $E\left(p_{0} p_{1} \ldots\right)$, welche z. B. Nevanlinna [5] beschrieben hat.

In ähnlicher Weise wie Nevanlinna [5, S. 154] für den Fall $N=2$ hat Midtbö bewiesen, dass die Menge $M\left(p_{0} p_{1} \ldots\right)$ dann und nur dann von der Kapazität Null ist, wenn die Reihe

$$
\sum_{\nu=1}^{\infty} N^{-v} \ln p_{\nu}
$$

divergent ist. Im folgenden nehmen wir an, dass die verallgemeinerten Cantor'schen Mengen, von denen wir Gebrauch machen, diese Bedingung erfüllen.

Für den Fall $N=2$ kann die Voraussetzung (9) durch die schwächere $p_{n}>1(n=1,2, \ldots)$ ersetzt werden (siehe af Hällström [4]).

7. Es sei nun der Rand unseres Gebietes $B$ eine Punktmenge der eben beschriebenen Art. Eine Ecke von $P_{0}$ möge z. B. in $z=0$ und eine Nachbarecke in $z=1$ liegen. Eine zugehörige Evans-Selbergfunktion hat Midtbö für den Fall $N=2$ gefunden (vgl. auch af Hällström [3][4]). Eine Verallgemeinerung der Midtbö'schen Funktion erhält man folgendermassen:

Wir belegen den Mittelpunkt jedes der $N^{n}$ Vielecke $P_{n}$ mit einer Masse von der Grösse $N^{-n}$ und betrachten das entsprechende logarithmische Potential in einem Punkte $z$ von $B$

$$
g_{n}(z)=N^{-n} \sum_{\nu=1}^{N^{n}} \ln \left(1 /\left|z-\xi_{n, v}\right|\right) .
$$


Hier ist $\xi_{n, v}$ der $\nu$ 'te Mittelpunkt. Wir werden nun zeigen, dass

$$
g(z)=\lim _{n \rightarrow \infty} g_{n}(z)
$$

die Eigenschaften einer Evans-Selbergfunktion hat (siehe Kap. I, Nr. 2).

Zuerst beweisen wir die beiden ersten Eigenschaften. Da $g_{n}(z)$ dieselben Eigenschaften hat $\left(g_{n}(z)\right.$ ist jedenfalls in einem gegebenen Punkte $z$ aus $B$ harmonisch, wenn $n$ genügend gross ist), ist es hinreichend zu zeigen, dass die Funktionenfolge $g_{n}(z)$ in jedem abgeschlossenen Teilgebiet von $B$ gleichmässig konvergiert.

Sei nun $\varepsilon$ eine beliebig kleine positive $Z$ ahl und $D$ ein abgeschlossenes Teilgebiet von $B$. Wir wählen $n_{\varepsilon}$ so gross, dass die Menge $M$ der Punkte sämtlicher $P_{n_{\varepsilon}}$ ausserhalb $D$ liegt, und ausserdem so gross, dass $d_{n_{\varepsilon}} / \delta<\varepsilon$ wird. Hier ist $\delta$ der Abstand des Randes des Gebietes $D$ von $M$. Wir bemerken, dass $g_{n+v}(z)-g_{n}(z)$ als eine durch $N^{n+v}$ dividierte Summe aus $N^{n+v}$ Ausdrücken der Form $\pm \ln (|z-\eta| /|z-\vartheta|)$ geschrieben werden kann $(|z-\eta| \geqq|z-\vartheta|)$. Die Punkte $\eta$ und $\vartheta$ gehören zu demselben $P_{n}$. Nun ist für $n \geqq n_{\varepsilon}$

$$
\ln (|z-\eta| /|z-\vartheta|) \leqq \ln (1+|\vartheta-\eta| /|z-\vartheta|)<\ln \left(1+d_{n_{\varepsilon}} / \delta\right)<d_{n_{\varepsilon}} / \delta<\varepsilon .
$$

Also gilt für alle $\nu \geqq 1$ und für jedes $z$ in $D$ die Ungleichheit

$$
\left|g_{n+\nu}(z)-g_{n}(z)\right|<\varepsilon,
$$

wenn $n \geqq n_{\varepsilon}$ ist, w. z. b. w. Die Konvergenz ist offenbar auch gleichmässig, wenn $D$ den unendlich fernen Punkt enthält.

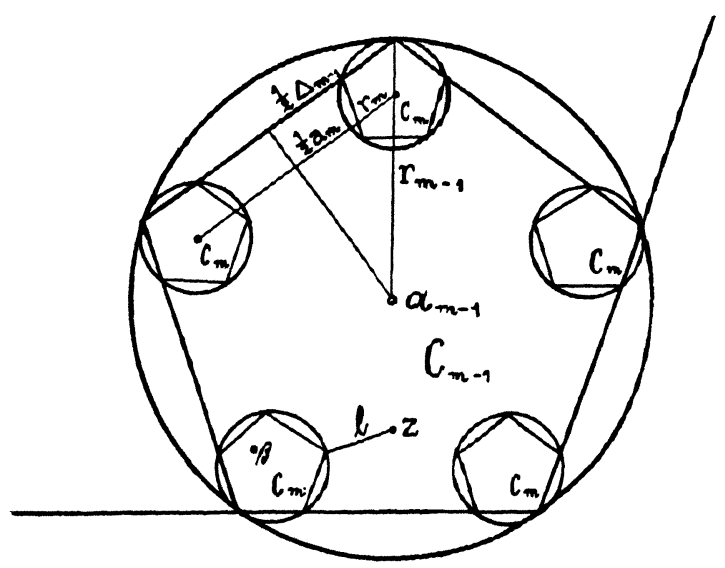

Fig. 2. 
Nachstehender Beweis für die dritte Eigenschaft ist, abgesehen von einigen Änderungen, der Midtbö'sche:

Sei $z$ ein Punkt in $B$, dessen Abstand $l$ von der Cantor'schen Punktmenge kleiner als ein gegebenes $d_{m}$ ist (siehe Fig. 2). Wir betrachten zuerst die Funktion $g_{n}(z)$. Hier sei $n \geqq m$ und ausserdem so gross, dass $z$ ausserhalb der $P_{n}$ liegt. Der nächste Cantor'sche Punkt gehört zu einem $C_{m}$. Das logarithmische Potential der Massenbelegung dieses Kreises ist in $z$ grösser als $-N^{-m} \ln \left(l+d_{m}\right)$. Das soeben erwähnte $C_{m}$ hat $N-1$ assoziierte $C_{m}$. Ihre Massenbelegung erzeugt in $z$ ein logarithmisches Potential grösser als

$$
-(N-1) N^{-m} \ln \left(l+d_{m-1}\right) .
$$

Die $N$ betrachteten $C_{m}$ liegen in einem $C_{m-1}$. Das der Masse auf den assoziierten $C_{m-1}$ entsprechende logarithmische Potential ist grösser als

u. s. w. Es wird also

$$
-(N-1) N^{-m+1} \ln \left(l+d_{m-2}\right)
$$

$$
g_{n}(z)>-N^{-m} \ln \left(l+d_{m}\right)-(N-1) N^{-m} \sum_{\nu=0}^{m-1} N^{v} \ln \left(l+d_{m-v-1}\right) .
$$

Ersetzen wir nun $l+d_{\mu}$ durch $2 \Delta_{\mu} / \sin (\pi / N)$, so erhalten wir $g_{n}(z)>-N^{-m} \ln \Delta_{m}-(N-1) N^{-m} \sum_{\nu=0}^{m-1} N^{m-v-1} \ln \Delta_{v}+\ln \sin (\pi / N)-\ln 2$.

Wegen $\Delta_{0}=1,1 / \Delta_{v}=2 p_{1} \cdot 2 p_{2} \cdot \ldots \cdot 2 p_{v}(v \geqq 1)$, ergibt sich

$$
\begin{aligned}
g_{n}(z)>N^{-m} \sum_{\mu=1}^{m} \ln \left(2 p_{\mu}\right)+(N-1) \sum_{\nu=1}^{m-1}\left\{N^{-v-1}\right. & \left.\sum_{\mu=1}^{\nu} \ln \left(2 p_{\mu}\right)\right\}+ \\
& +\ln \sin (\pi / N)-\ln 2 .
\end{aligned}
$$

Nun ist

$$
\begin{aligned}
\sum_{\nu=1}^{m-1}\left\{N^{-\nu-1} \sum_{\mu=1}^{\nu} \ln \left(2 p_{\mu}\right)\right\} & =\sum_{\mu=1}^{m-1}\left\{\ln \left(2 p_{\mu}\right) \sum_{\nu=\mu}^{m-1} N^{-\nu-1}\right\} \\
& =\sum_{\mu=1}^{m-1} \ln \left(2 p_{\mu}\right)(N-1)^{-1}\left(N^{-\mu}-N^{-m}\right) .
\end{aligned}
$$

Schliesslich haben wir also

woraus sich

$$
g_{n}(z)>\sum_{\mu=1}^{m} N^{-\mu} \ln \left(2 p_{\mu}\right)+\ln \sin (\pi / N)-\ln 2,
$$

$$
g(z) \geqq \sum_{\mu=1}^{m} N^{-\mu} \ln \left(2 p_{\mu}\right)+c \quad\left(l<d_{m}\right)
$$


ergibt. Hier ist

Da die Reihe

$$
c=\ln \sin (\pi / N)-\ln 2 .
$$

$$
\sum_{\mu=1}^{\infty} N^{-\mu} \ln \left(2 p_{\mu}\right)
$$

divergent ist, haben wir also auch die Eigenschaft $3^{\circ}$ für $g(z)$ nachgewiesen.

Bemerkung. Die Funktion $g(z)$ lässt sich offenbar als eine Summe von $N^{m}$ Gliedern schreiben. Jeder Summand ist dann der Grenzwert desjenigen Teiles von $g_{n}(z)(n \geqq m)$, der das logarithmische Potential im Punkte $z$ der Massenbelegung eines bestimmten $C_{m}$ ist. Wir werden diesen Summanden den dem eben erwähnten Kreise entsprechenden nennen.

§ 2. Eine hinreichende Bedingung dafür, dass $g(z)$ in dem Mittelpunkte eines $\boldsymbol{C}_{m-1}$ kleiner ist als in den zu diesem Kreise gehörigen $\boldsymbol{C}_{m}$

8. In Fig. 2 ist der Mittelpunkt $\alpha_{m-1}$ eines Kreises $C_{m-1}$ eingezeichnet, und $\beta$ ist ein beliebiger Punkt eines der Kreise $C_{m}$ in diesem $C_{m-1}$. Es werde angenommen, dass $\beta$ zu $B$ gehört.

Zuerst werden wir eine untere Schranke für den kleinsten Abstand $a_{m}$ zwischen zwei assoziierten $C_{m}(m \geqq 1)$ finden. Aus der Figur 2 ergibt sich

$$
a_{m}=\Delta_{m-1}\left(1-1 /\left(2 p_{m}\right)\right)-2 r_{m}>\frac{1}{2} \Delta_{m-1}-d_{m} .
$$

Nun ist der Summand von $g(z)$, welcher demjenigen Kreise $C_{m}$ entspricht, $\operatorname{der} \beta$ enthält, in diesem Punkte mindestens gleich $-N^{-m} \ln d_{m}$, und die den assoziierten $C_{m}$ entsprechenden Glieder der Funktion sind zusammen mindestens gleich $-(N-1) N^{-m} \ln d_{m-1}$. Ferner ist die Summe aller genannten Summanden in $\alpha_{m-1}$ höchstens gleich

$$
-N^{-m+1} \ln \left(r_{m-1}-d_{m}\right) \text {. }
$$

Haben nun die Werte der übrigen Glieder von $g(z)$ in $\alpha_{m-1}$ und in $\beta$ die Differenz $U$, und ist

$$
\ln \left(2 p_{m}\right)-N \ln d_{m-1}>-N \ln \left(r_{m-1}-d_{m}\right)+N^{m} U,
$$

so wird also $g(z)$ in $\beta$ grösser als in $\alpha_{m-1}$.

Wir werden zuerst die Grösse $U$ abschätzen. Die Differenz zwischen den Werten in $\alpha_{m-1}$ und in $\beta$ des logarithmischen Potentials einer Masse $M$ im Abstand $\delta$ von $\alpha_{m-1}$ ist höchstens gleich

$$
M \ln \left(1+\left|\alpha_{m-1}-\beta\right| / \delta\right) \text {. }
$$


Nun hat das $C_{m-1}$ der Figur $N-1$ assoziierte $C_{m-1}$. Der Teil von $U$, welcher diesen Kreisen entspricht, ist dann höchstens gleich

$$
(N-1) N^{-m+1} \ln \left(1+r_{m-1} / a_{m-1}\right) \text {. }
$$

Alle eben genannten $C_{m-1}$ gehören zu demselben $C_{m-2}$, und dieses hat wieder $N-1$ assoziierte $C_{m-2}$. Dementsprechend erhält $U$ einen Beitrag, welcher höchstens gleich

$$
(N-1) N^{-m+2} \ln \left(1+r_{m-1} / a_{m-2}\right)
$$

ist, u. s. w. Also ergibt sich für $m \geqq 2$

$$
N^{m} U \leqq \sum_{\nu=1}^{m-1}(N-1) N^{v} \ln \left(1+r_{m-1} / a_{m-\nu}\right) .
$$

Um diese Abschätzung von $U$ vereinfachen zu können, suchen wir eine hinreichende Bedingung dafür, dass

$$
a_{m-p}>r_{m-1} N^{v+1}
$$

für $1 \leqq \nu \leqq m-1$ ist. Wir nehmen zunächst an, dass $2 p_{n}>N(n \geqq 1)$ ist. Dann wird (14) richtig, wenn $a_{m-\nu}>r_{m-\nu} N^{2}$ ist. Wegen (11) muss dies sicher gelten, wenn $d_{m-\nu-1} \sin (\pi / N)-2 d_{m-v}>d_{m-v} N^{2}$ erfüllt ist. $D a-$ her wird im folgenden

$$
2 p_{n}>\left(N^{2}+2\right) / \sin (\pi / N) \quad(n \geqq 1)
$$

vorausgesetzt, so dass (14) stattfindet. (Wegen (15) werden (9) und die Voraussetzung $2 p_{n}>N$ von selbst erfüllt.) Aus (13) und (14) ergibt sich, dass $N^{m} U<(m-1) \ln e$ für $m \geqq 2$ gilt.

Hiernach wird (12) sicher erfüllt, wenn

$$
\ln \left(2 p_{m}\right)>-N \ln \left(\frac{1}{2}-1 /\left(2 p_{m}\right)\right)+m-1
$$

ist. Da $2 p_{m}>6$ wegen (15), wird also

$$
2 p_{m} \geqq 3^{N} e^{m-1} \quad(m \geqq 2)
$$

eine der Überschrift dieses Paragraphen entsprechende Bedingung.

Wir wissen nicht, ob alle $p_{m}(m \geqq 2)$ der Ungleichheit (16) genügen; aber wegen der Divergenz der Reihe

$$
\sum_{\nu=1}^{\infty} N^{-v} \ln p_{\nu}
$$

gilt diese Beziehung für unendlich viele $m$. 


\section{§ 3. Abschätzung von $F(\lambda)$}

9. Sei $p_{m}$ eine der Grössen $p_{v}$, für welche (16) richtig ist $(m \geqq 2)$. Wir betrachten die Werte von $g(z)$ in den Mittelpunkten der Kreise $C_{m-1}$. Den kleinsten dieser Werte bezeichnen wir mit $\lambda^{\prime}$. Die Niveaukurve $G_{\lambda^{\prime}}$ (siehe Nr. 2) zerteilt kein $C_{m}$, da $g(z)$ in diesen Kreisen grösser als $\lambda^{\prime}$ ist. Nun gibt es nach Nr. 2 im Inneren eines geschlossenen Teiles dieser Kurve mindestens einen Cantorpunkt. Wir erhalten daher (siehe (5)) $n_{G}\left(\lambda^{\prime}\right) \leqq N^{m}-1$. Da $\lambda^{\prime}$ der Wert von $g(z)$ in einem Punkte ist, dessen Abstand vom nächsten Cantorpunkt kleiner als $d_{m-1}$ ist, wird nach (10)

Hier ist

$$
\lambda^{\prime} \geqq \sum_{\nu=1}^{m-1} u_{\nu}+c
$$

$$
u_{p}=N^{-v} \ln \left(2 p_{v}\right) .
$$

Nun muss jede Niveaukurve von $g(z)$, welche aus mindestens zwei geschlossenen Teilen besteht, mit $C_{0}$ Punkte gemeinsam haben, weil Cantorpunkte im Inneren jedes Teiles liegen. Da $g(z)$ in $C_{0}$ mindestens gleich $\ln \sin (\pi / N)$ ist, muss $n_{G}(\lambda)$ für $\lambda<c$ verschwinden.

Wir haben also gemäss (5)

SATz 1. Besteht die Beziehung (15), sind $m_{1}+1<m_{2}+1<\ldots<m_{n+1}+1$ die ersten $n+1$ Indizes, für welche (16) gilt $\left(m_{1} \geqq 1\right)$, und setzen wir $m_{0}=0$, so gilt für $\lambda$ im Intervalle $\left[\lambda_{m_{n}}, \lambda_{m_{n+1}}\right]$

$$
F(\lambda)<\sum_{\mu=1}^{n}\left\{N^{m_{\mu}+1} \sum_{\nu=m_{\mu-1}+1}^{m_{\mu}} u_{\nu}\right\}+\left(\lambda-\lambda_{m_{n}}\right) N^{m_{n+1}+1}-2 \lambda+K .
$$

Hier ist

\section{Speziell erhalten wir}

$$
\lambda_{k}=\sum_{\nu=1}^{k} u_{\nu}+c
$$

SATz 2. Ist für alle $m \geqq 2$ die Beziehung (16) erfüllt, so gilt für $\lambda$ im Intervalle $\left[\lambda_{n}, \lambda_{n+1}\right]$

$$
F(\lambda)<N \sum_{\nu=1}^{n} \ln \left(2 p_{\nu}\right)+\left(\lambda-\lambda_{n}\right) N^{n+2}-2 \lambda+K .
$$

Für $N=2$ ergibt ein anderes Verfahren die bessere Abschätzung

$$
F(\lambda)<\sum_{\nu=1}^{n} \ln \left(2 p_{\nu}\right)+\left(\lambda-\lambda_{n}\right) 2^{n+1}-2 \lambda+K .
$$

Setzen wir in dem ersten Satze 


$$
R(\lambda)=\sum_{\mu=1}^{n}\left\{N^{m_{\mu}+1} \sum_{\nu=m_{\mu-1+1}}^{m_{\mu}} u_{v}\right\}+\left(\lambda-\lambda_{m_{n}}\right) N^{m_{n+1}+1},
$$

wenn $\lambda$ im Intervalle $\left[\lambda_{m_{n}}, \lambda_{m_{n+1}}\right]$ liegt, so wird

$$
F(\lambda)<R(\lambda)-2 \lambda+K \text {. }
$$

\section{§ 4. Die Ordnung oder Hyperordnung von $\boldsymbol{R}(\lambda)$}

10. Die Ordnung einer positiven, monoton zunehmenden Funktion $\varphi(\lambda)$ von $\lambda$ wird als

$$
s=\limsup _{\lambda \rightarrow \infty}\left(\lambda^{-1} \ln \varphi(\lambda)\right)
$$

definiert. Ist die Ordnung unendlich, so wird eine Hyperordnung

$$
\sigma=\limsup _{\lambda \rightarrow \infty}\left(\lambda^{-1} \ln \ln \varphi(\lambda)\right)
$$

eingeführt (siehe [2, S. 21]).

Vor der Bestimmung der Ordnung oder Hyperordnung von $R(\lambda)$ zeigen wir, dass

$$
u_{\nu} \geqq v^{-1}, \quad \nu=1,2, \ldots,
$$

eine hinreichende Bedingung für die Gültigkeit von (15) ist. Wegen $N^{v} / v \geqq N, v=1,2, \ldots$, ist die Behauptung sicher richtig, falls

$$
e^{N} \sin (\pi / N)>N^{2}+2
$$

gilt. Nun ist $\sin x / x \geqq 2 / \pi(0 \leqq x \leqq \pi / 2)$, und integriert man die für alle positiven $y$ gültige Ungleichheit $e^{y}>1+3 y^{2} / 2$ von 0 bis $y$, so folgt $e^{y}>y+y^{3} / 2$. Also ergibt $\operatorname{sich} e^{N} \sin (\pi / N)>N^{2}+2$.

Es ist auch leicht $\mathrm{zu}$ bestätigen, dass (23) die Ungleichheit

$$
\ln \left(2 p_{v}\right) \geqq N \ln 3+v-1
$$

für $v \geqq 5(N=2)$, oder für $v \geqq 2(N \geqq 3)$, zur Folge hat.

Im folgenden setzen wir die Gültigkeit von (23) voraus.

11. Liegt nun $\lambda$ im Intervalle $\left[\lambda_{n}, \lambda_{n+1}\right]\left(\lambda_{n}>0, n \geqq 4\right)$, so haben wir

$$
\lambda^{-1} \ln R(\lambda)<\lambda^{-1} \ln \left[N^{n+2}(\lambda-c)\right] \leqq \lambda_{n}^{-1}(n+2) \ln N+\lambda^{-1} \ln (\lambda-c)
$$

und gleichzeitig

$$
\lambda^{-1} \ln R(\lambda)>\lambda_{n+1}{ }^{-1} \ln \left(N^{n+1} n^{-1}\right) .
$$

Aus diesen Ungleichheiten entnimmt man den 
SATZ 3. Wenn $u_{n} \geqq n^{-1}(n=1,2, \ldots)$ ist, so ist die Ordnung von $R(\lambda)$ gleich

$$
\limsup _{n \rightarrow \infty}\left(\lambda_{n}{ }^{-1} n\right) \ln N \text {. }
$$

Ist also $u_{n}=t^{n}(t>1)$, so hat $R(\lambda)$ die Ordnung Null, und dasselbe gilt, wenn $u_{n}=1+(n-1) d(d>0)$ gesetzt wird. Ferner entspricht $u_{n}=k+n^{-1}$ der Ordnung $k^{-1} \ln N$ von $R\left(\lambda_{2}\right)$.

12. Wird die Ordnung von $R(\lambda)$ unendlich, so bestimmen wir die Hyperordnung. In ähnlicher Weise wie in $\mathrm{Nr} .11$ beweist man den

SATz 4. Wenn $u_{n} \geqq n^{-1}(n=1,2, \ldots)$ ist, so ist die Hyperordnung von $R(\lambda)$ gleich

$$
\limsup _{n \rightarrow \infty}\left(\lambda_{n}{ }^{-1} \ln n\right) \text {. }
$$

Setzen wir $u_{n}=k n^{-1}(k \geqq 1)$, so wird folglich die Hyperordnung von $R(\lambda)$ gleich $k^{-1}$, da in diesem Falle $\ln (n+1)<\left(\lambda_{n}-c\right) / k<1+\ln n$ gilt.

Wegen $u_{n} \geqq n^{-1}$ kann die Hyperordnung von $R(\lambda)$ nicht grösser als 1 sein.

\section{§ 5. Die Defektrelation}

13. Zum Abschluss dieser Arbeit sollen einige hinreichende Bedingungen für die Gültigkeit der Defektrelation (8) gegeben werden.

Sei zuerst vorausgesetzt, dass $s=\lim \sup _{n \rightarrow \infty}\left(\lambda_{n}{ }^{-1} n\right) \ln N$ endlich ist. Aus (4) folgt

$$
\sum_{i=1}^{q} \frac{m\left(\lambda, w_{i}\right)}{T(\lambda)}+\frac{N_{1}(\lambda)}{T(\lambda)}<2+\frac{F(\lambda)}{T(\lambda)}+\frac{O(\ln T(\lambda))}{T(\lambda)},
$$

ausser vielleicht auf einer Intervallfolge $I_{1}(\lambda)$, auf welcher $\int T(\lambda) d \lambda$ endlich ist.

Ist die Ordnung von $T(\lambda)$ grösser als $s_{1}=s+2 \varepsilon(\varepsilon>0)$, so gilt nach einem Hällström'schen Hilfssatz [2, S. 62] auf einer Intervallfolge $I(\lambda)$, auf welcher $\int e^{\lambda} d \lambda$ unendlich ist, die Ungleichheit

$$
T(\lambda)>e^{\lambda_{1}} \quad(\lambda>0) .
$$

Es ist leicht zu zeigen, dass sogar stets eine Intervallfolge $I_{2}(\lambda)$ von unendlicher Gesamtlänge existiert, auf der (24) richtig ist.

Aus $I_{2}(\lambda)$ entfernt man den Durchschnitt von $I_{1}(\lambda)$ und $I_{2}(\lambda)$. Die übriggebliebene Intervallfolge $I_{3}(\lambda)$ ist dann auch von unendlicher Gesamtlänge. Nach dem dritten Satze, (22) und (24) wird nun für genügend grosse $\lambda$ auf dieser Intervallfolge $F(\lambda) / T(\lambda)<e^{\lambda\left(s_{1}-\varepsilon\right)} / e^{\lambda s_{1}}=e^{-\lambda \varepsilon}$. Also streben auf $I_{3}(\lambda)$ sowohl $F(\lambda) / T(\lambda)$ als $O(\ln T(\lambda)) / T(\lambda)$ mit wachsendem $\lambda$ 
gegen Null. Dann ist aber der untere Limes der linken Seite von (4a) höchstens gleich 2 , und wir haben somit den

SATZ 5. Sei $N^{-n} \ln \left(2 p_{n}\right) \geqq n^{-1}(n=1,2, \ldots)$. Ist dann

$$
s=\limsup _{n \rightarrow \infty}\left(\lambda_{n}{ }^{-1} n\right) \ln N
$$

endlich, und hat $f(z)$ eine der Midtbö'schen Potentialfunktion entsprechende Ordnung ${ }^{2}$ grösser als $s$, so gilt die Defektrelation.

Das obige Verfahren entspricht dem Hällström'schen [2, S. 63].

Dass es in $B$ meromorphe Funktionen $f(z)$ gibt, deren Ordnung grösser als $s$ ist, wird folgendermassen gezeigt:

Wir konstruieren eine Funktion $f(z)$, die in $B$ eindeutig und meromorph ist und in diesem Bereiche Nullstellen hat, welche eine gewisse isolierte Punktmenge $(b)$ bilden (siehe Osgood [7, S. 548]). Der unendlich ferne Punkt soll nicht zu $(b)$ gehören. Wir legen zuerst in $B$ eine Punktmenge, die Häufungspunkte in sämtlichen Cantorpunkten hat, und sonst nirgends (siehe [7, S. 551]). Die übrigen $b$-Punkte werden in folgender Weise gewählt: Sei $S=s+2 \varepsilon(\varepsilon>0)$. Falls es im Gebiete $\lambda<\lambda_{n}$ nicht schon so viele Nullstellen von $f(z)$ gibt, dass

$$
\int_{-\infty}^{\lambda_{n}} n(g, 0) d g>e^{\lambda_{n} S}
$$

ist (siehe (2)), so werden im Bereiche $\lambda_{n-1} \leqq \lambda<\lambda_{n}$ so viele Punkte hinzugefügt, dass die Ungleichheit erfüllt wird. Dieser Prozess wird sukzessiv für $n=2,3, \ldots$ ausgeführt, wonach $N\left(\lambda_{n}, 0\right)>e^{\lambda_{n}(s+\varepsilon)}$ für alle genügend grossen $\lambda_{n}$ gilt. Da nach (3) $T(\lambda) \geqq N(\lambda, 0)$ ist, muss also die Ordnung von $T(\lambda)$ grösser als $s$ sein.

14. Hiernach werde angenommen, dass $R(\lambda)$ keine endliche Ordnung besitzt. Nun ist die Hyperordnung einer Funktion $\varphi(\lambda)$ gleich der Ordnung von $\ln \varphi(\lambda)$. Ist also die Hyperordnung von $T(\lambda)$ grösser als

$$
\sigma>\limsup _{n \rightarrow \infty}\left(\lambda_{n}^{-1} \ln n\right),
$$

so gilt nach dem in Nr. 13 zitierten Hilfssatze auf einer Intervallfolge $I(\lambda)$ von unendlicher Gesamtlänge

$$
T(\lambda)>e^{e \lambda \sigma} \quad(\lambda>0) .
$$

2 Man sagt, die Funktion $f(z)$ habe dieselbe Ordnung wie ihre charakteristische Funktion. 
Mit Benutzung des vierten Satzes, (22) und (25) erhalten wir nach einem ähnlichen Verfahren wie früher den

SAtz 6. Sei $N^{-n} \ln \left(2 p_{n}\right) \geqq n^{-1}(n=1,2, \ldots)$. Hat dann $f(z)$ eine der Midtbö'schen Potentialfunktion entsprechende Hyperordnung grösser als

so gilt die Defektrelation.

$$
\limsup _{n \rightarrow \infty}\left(\lambda_{n}{ }^{-1} \ln n\right),
$$

In ähnlicher Weise wie in Nr. 13 lässt sich zeigen, dass es in $B$ Funktionen von einer Hyperordnung grösser als

$$
\limsup _{n \rightarrow \infty}\left(\lambda_{n}{ }^{-1} \ln n\right)
$$

gibt.

$$
n \rightarrow \infty
$$

\section{LITERATUR}

1. G. Evans, Potentials and positively infinite singularities of harmonic functions, Monatsh. Math. Phys. 43 (1936), 419-424.

2. G. af Hällström, Über meromorphe Funktionen mit mehrfach zusammenhängenden Existenzgebieten, Acta Acad. Aboensis, XII: 8 (1939).

3. G. af Hällström, Zur Berechnung der Bodenordnung oder Bodenhyperordnung einer eindeutigen Funktion, Ann. Acad. Sci. Fenn. AI, Nr. 193 (1955).

4. G. af Hällström, On the capacity of generalized Cantor sets, Acta Acad. Aboensis, XX:5 (1955).

5. R. Nevanlinna, Eindeutige analytische Funktionen, 2 Aufl., Berlin, 1953.

6. K. Noshiro, Contributions to the theory of the singularities of analytic functions, Jap. J. Math. 19 (1948), 299-327.

7. W. F. Osgood, Lehrbuch der Funktionentheorie I, 4. Aufl., Leipzig und Berlin, 1923.

8. H. Selberg, Uber die ebenen Punktmengen von der Kapazität Null, Avh. Norske Vid. Akad. Oslo, I, 1937, Nr. 10, 1-10.

9. M. Tsuji, On the behavior of a meromorphic function in the neighbourhood of a closed set of capacity zero, Proc. Imp. Acad. Tokyo 18 (1942), 213-219.

10. M. Tsuji, Theory of meromorphic functions in a neighbourhood of a closed set of capacity zero, Jap. J. Math. 19 (1944), 139-154. 